\title{
The depressive symptoms are moderators of cognitive and functional performances in normal and pathological aging?
}

Os sintomas depressivos são moderadores dos desempenhos cognitivo e funcional no envelhecimento normal e patológico?

Jonas Jardim de Paula

Programa de Pós-Graduação em Neurociências, Universidade Federal de Minas Gerais (UFMG), Belo Horizonte MG, Brazil. 


\section{THESES}

\section{ABSTRACT}

Depression (DEP) is usually associated with cognitive and functional impairment in the elderly. The present study aimed at validating a neuropsychological assessment protocol for low-educated elderly patients and at investigating the role of DEP as a moderator of cognitive impairment in participants $(n=274)$ without dementia, amnestic mild cognitive impairment $(M C l)$, and mild Alzheimer's disease (AD). Through an integrative review of the literature and subsequent validation, the Geriatric Depression Scale (GDS-15) was selected as a DEP measure. A multidimensional neuropsychological exam protocol composed by tests adapted or created for the assessment of executive functions, language, memory, visuospatial abilities, and functionality in low-educated elderly patients was validated by different methods in this study. DEP showed a moderating effect on cognitive performance of elderly people without dementia, with larger effect sizes on memory and executive functions and it was related to functional impairment. However, the phenomenon is less expressive in AD and MCl. A secondary analysis suggested that executive dysfunction mediates the deficit in other cognitive functions. The study validated a neuropsychological exam protocol for the low-educated Brazilian elderly population. The result suggests that DEP moderated cognitive performance and this effect may be mediated by executive dysfunction.

Key words: depression, cognitive impairment, Geriatric Depression Scale. 